strategy to increase the donor pool. Long-term outcomes in recipients of ECD livers have not been well studied. We studied the characteristics, utilization and recipient outcomes of ECDs.

Methods We retrospectively studied consecutive adults who underwent deceased LT between 2006-2018. Donor and recipient data at LT and recipient outcomes were collected from a prospective database. ECD was defined using Eurotransplant criteria as any of these donor characteristics: age $>65$ years, ventilation $>7$ days, BMI $>30 \mathrm{~kg} / \mathrm{m} 2$, liver steatosis $>40 \%$, sodium $>165 \mathrm{mmol} / \mathrm{L}$, ALT $>105 \mathrm{U} / \mathrm{L}$ or AST $>90$ $\mathrm{U} / \mathrm{L}$, and bilirubin $>51 \mathrm{umol} / \mathrm{L}$. The primary outcome of interest was graft survival (time to retransplantation or death). Results During the study period (median follow-up 50.6 months), 739 donors were utilized for LT. Of these, 432 (58.4\%) were ECDs. Corresponding to the definition, ECDs were older (51vs.47years), with higher BMI (26.4vs.24.5 kg/ $\mathrm{m}^{2}$ ), transaminases (ALT $42 \mathrm{vs} .24 \mathrm{U} / \mathrm{L}$ ) and graft steatosis (11.4\%vs.0\% $\geq$ S2) compared to non-ECDs (median values, all $P<0.001$ ). ECDs also had a worse renal function (creatinine $80 \mathrm{vs.67}$ umol/L, $P<0.001)$ and a higher donor risk index (Feng et al. 2006) (1.65vs.1.52, $P<0.001)$. There were no differences in recipient characteristics of ECD vs. non-ECD grafts except ECD recipients were less likely receive split grafts (10.9\%vs.18.9\%, $P=0.002$ ). The proportion of ECDs among utilized grafts did not change over time. 157 patients experienced graft loss during follow-up (31 retransplants, 126 deaths). ECDs had similar long-term graft survival compared to non-ECDs, although outcomes appeared to be worse when $\geq 3$ criteria were met (figure1A-B). No individual ECD criteria were predictive for poorer graft survival. Among patients transplanted for HCC, ECD vs. non-ECD grafts resulted in similar recurrence-free survival (figure 1C).

Conclusions ECD livers meeting up to 2 Eurotransplant criteria can be safely used without impacting long-term graft survival. This has implications for organ utilization.

\section{IDDF2019-ABS-0222 EXCHANGE TRANSFUSION IN NEONATAL HYPERBILIRUBINEMIA AND BILIRUBIN ENCEPHALOPATHY: A LONG WAY TO GO}

Mamta Jajoo*. Chacha Nehru Bal Chikitsalaya,(Associated to MAulana Azad Medical College)Delhi, India

\subsection{6/gutjnl-2019-IDDFabstracts.290}

Background Hyperbilirubinemia is the most common morbidity in neonates. Exchange transfusion (ECT) has an important role in the prevention of Bilirubin Encephalopathy in neonates.

Aim To evaluate the incidence and risk factors of Bilirubin Encephalopathy in neonates presenting with neonatal hyperbilirubemia requiring ECT.

Subjects All neonates admitted with hyperbilirubinemia requiring exchange transfusion.

Methods Retrospective study underwent exchange transfusion over a period of 30 months in a level III extramural Neonatal Intensive care Unit of Delhi. Demographic data, etiology of jaundice, the presence of encephalopathy, details of ECT and ECT-related adverse events were analyzed.

Results Total of 1675 neonates were admitted, out of which 136(8.1\%) neonates underwent exchange transfusion. The mean(SD) gestational age and mean weight(SD) on admission were $36 \pm 3$ weeks and $2144.5 \pm 678$ gram respectively. The mean age of presentation was 5.48 \pm 3.9 days and the mean day on which jaundice appeared was $4.9 \pm 3.33$ days.

The mean bilirubin on presentation and duration of phototherapy were $24.2 \pm 5.8 \mathrm{mg} / \mathrm{dl}$ and $3.2 \pm 1.7$ days respectively. Out of 136 neonates,20(14.7\%) were home delivered. Sepsis $49(36 \%)$ was the most common cause of $\mathrm{NNH}$ requiring ECT. Other causes were ABO incompatibility(21.3\%), Rh incompatibility(11.7\%), hypothyroidism(3.6\%),G6PD deficiency $(0.7 \%)$ and Idiopathic . Five (3.6\%) neonates were blood culture positive. ECT through umbilical route was done in 115 $(84.5 \%)$ and through peripheral route in 21 (15.4\%). More than one ECTs were required in 5(3.6\%). The complications of ECT were hypocalcaemia (38.2\%), thrombocytopenia $(17.6 \%)$ and hypothermia (11\%). Thirty-nine neonates (28.6\%) had encephalopathy on admission; the mean day of presentation, weight and bilirubin in these neonates were 5.5 \pm 3.7 days, $2142.6 \pm 763$ grams, $26.9 \pm 6.13 \mathrm{mg} / \mathrm{dl}$ respectively.

Conclusions The incidence of $\mathrm{NNH}$ developing Bilirubin encephalopathy is still very high in our country, most probably because of early discharge, loss to follow up and delay in diagnosis and treatment. Timely intervention and proper counseling of mother at the time of discharge after birth is very important to prevent encephalopathy in neonates with $\mathrm{NNH}$

\section{IDDF2019-ABS-0237 CLINICO-ETIOLOGICAL PROFILE OF HEPATOCELLULAR CARCINOMA AT A TERTIARY HOSPITAL IN COASTAL INDIA}

Ravella Ranjith*, Nishitha Shetty, Prashanth YM, Dinesh Shet. Father Muller Medical College and Hospital, India

\subsection{6/gutjn|-2019-IDDFabstracts.291}

\section{Background}

- Hepatocellular carcinoma (HCC) is a primary malignancy of the liver and is apparent as the main cause of death in patients with liver cirrhosis or chronic hepatitis $\mathrm{B}$ or $\mathrm{C}$ virus infection. It is emerging as a major leading cause of cancer mortality.

- In India, the mean incidence of Hepatocellular carcinoma is $2.77 \%$ for males and $1.38 \%$ for females based on the population registry.

- Our study aims to assess the clinico-etiological profile of HCC at a tertiary hospital in India.

Methods

- Cross-sectional, observational and hospital-based retrospective study. Patients diagnosed as HCC from Jan 2014 to Feb 2019, registered in Hepatocellular Cancer Registry and admitted to the hospital, were reviewed.

- Clinical, biochemical, serological and radiological details were noted from case records and analysed.

Abstract IDDF2019-ABS-0237 Table 1

\begin{tabular}{ll}
\hline Parameter & Number of patients (\%) \\
\hline Right Lobe Involvement & $34(64.15 \%)$ \\
Left Lobe Involvement & $03(5.66 \%)$ \\
Bilobar Involvement & $16(30.18 \%)$ \\
\hline
\end{tabular}

